\title{
Studies of timing properties of SiPMs at Fermilab.
}

\section{Anatoly Ronzhin}

Fermi National Accelerator Laboratory

Kirk\&Pine, Batavia, Il 60502, US

E-mail: ronzhinefnal.gov

We continue FNAL program to study timing properties of silicon photomultipliers (SiPms). The used technique, i.e., DRS4 and ORTEC readout has 2-5 ps "electronic" time resolution. We have measured the single photoelectron time resolution (SPTR) for different silicon photomultipliers (SiPMs) size. The dependence of the SPTR on overvoltage presented. The shape of the SiPms signals was investigated and was changed according to timing requirements. We used the PiLas laser blue $(405 \mathrm{~nm})$ and red $(635 \mathrm{~nm})$ heads for this study. One of the goal was to investigate the performance of the DRS4 waveform digitizer for fast time measurements with the SiPMs. The SiPms with Cherenkov radiators were tested at $120 \mathrm{GeV}$ proton beam at Fermilab. Time resolution of 14 ps per SiPM was obtained. A few algorithms, fitting to the best time resolution were investigated. With the DRS4 readout we did not find a significant difference in the time resolution with and without a clipping capacitance when the light pulse is very short (PiLas or Cherenkov light) [1-3].

International Workshop on New Photon-detectors,

LAL Orsay, France

June 13-15, 2012 


\section{Introduction}

\subsection{Main SiPM timing parameters}

Let's introduce a few terms with respect to SiPM's timing.

SiPM Signal Shape, (SSS), ( $\mathrm{Tr}$ - signal rise time, Tf - signal fall time; $\mathrm{Tr}$ is often measured at $10 \%-90 \%$ of the signal amplitude; Single Photoelectron Time Resolution (SPTR); Signal (S) and Noise $(\mathrm{N}),(\mathrm{S}$ and $\mathrm{N}$ are in number of electrons, $\mathrm{mV}$, etc.).

The formula for detector time resolution (TR) is:

$$
\sigma=\operatorname{Tr} \times 1 /(\mathrm{S} / \mathrm{N})
$$

$\sigma$ is sigma of fitting Gaussian distribution, $\mathrm{Tr}$ - signal rise time, $\mathrm{S} / \mathrm{N}$ is signal to noise ratio. Time resolution (TR) is a time jitter of the measured time interval between two signals. These two signals are named as "start" and "stop". TR depends on all of the above parameters, including parameters which named "time stamps". Time stamp is chosen point on the signal. This point should have the smallest timing fluctuation. The time stamp depends also on used front end (FE) electronics, e.g. leading edge discriminator (LED), constant fraction discriminator (CFD). Currently waveform digitizers used for timing analysis. The digitizers allow off line signal analysis a contrary to FE above where we have to make decision at the level of data taking. Different algorithm applied to signal timing analysis with the digitizers (DRS4, PSEC4, etc.) also results with different TR.

\subsection{SiPM Signal Shape, SSS}

It is well established that SiPM signal shape does not depends significantly on numbers of detected photons, $\mathrm{N}_{\mathrm{ph}}$, when SiPM illuminated by short light pulse $\left(\mathrm{N}_{\mathrm{ph}}<<\right.$ $\mathrm{N}_{\mathrm{c}}$ ). $\mathrm{N}_{\mathrm{c}}$ is number of SiPM's cells. SiPM signal consists of 3 main parts (Fig. 1): rise time, recovery time and fast spike on leading edge in presence of parasitic capacitance. Rise time is mostly relates with avalanche development in SiPM, recovery time manages by diode charge through quenching resistor and fast spike on the signal leading edge depends on diode's parasitic capacitance value.

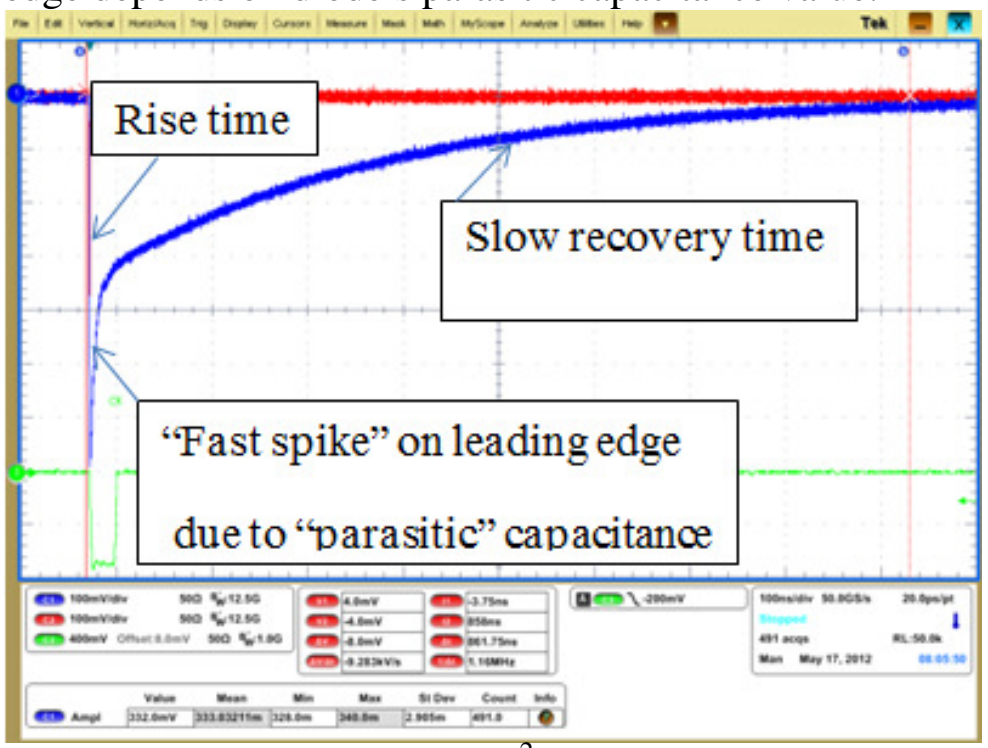

Fig. 1. STM trace, $3.5 \times 3.5 \mathrm{~mm}^{2} .3,600$ cells. $\mathrm{N}$ on P structure. PiLas light. 
Fast oscilloscope (50 GS/s, 20 ps sampling) and PiLas laser used for SSS measurement. The PiLas laser light pulse is $\sim 33$ ps, FWHM. The PiLas equipped with the two heads, $405 \mathrm{~nm}$ and $635 \mathrm{~nm}$ of the wave length and allows change the light intensity by using TUNE. A library of traces of all tested SiPMs: STM, MPPC, SensL, CPTA, KETEK, FBK-IRST, MRS, etc. The library is used for SiPM output signal modeling with different light sources.

\subsection{Single Photoelectron Time Resolution, (SPTR).}

If we know the SPTR we can estimate the time resolution for some number of photoelectrons $\mathrm{N}_{\text {phes }}$ by using the formula:

$$
\sigma=\mathrm{SPTR} / \sqrt{ } \mathrm{N}_{\text {phes }}
$$

We have to note that the formula is only true when the light pulse shape does not change with the number of the photons increase, i.e. the number of the photoelectrons enhances due to light density (and not to light pulse duration).

The time distribution (SPTR) of the SiPM signals originated by single photon relative to trigger signal was measured. It is easy to identify single photoelectron by SiPM. We have to clip the SiPMs signal when measuring the SPTR with Ortec 9327 constant fraction discriminator, Fig.2. Fig. 3 shows the effect of clipping capacitance on SSS.

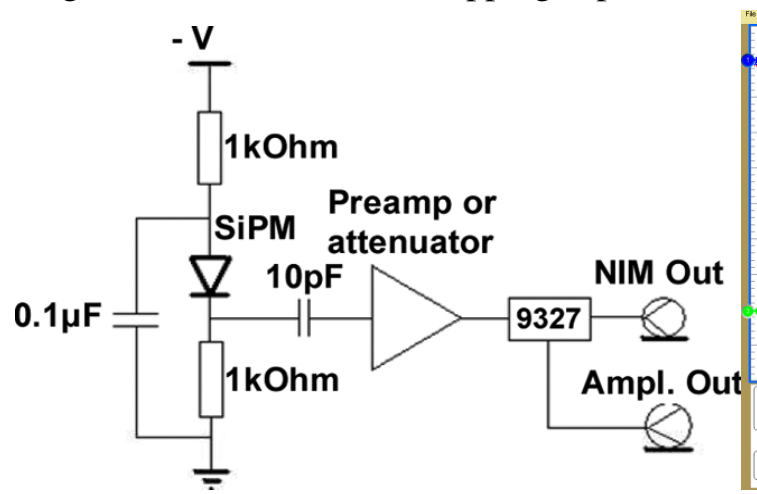

Fig. 2. Schematic of the SiPM clipping circuit.

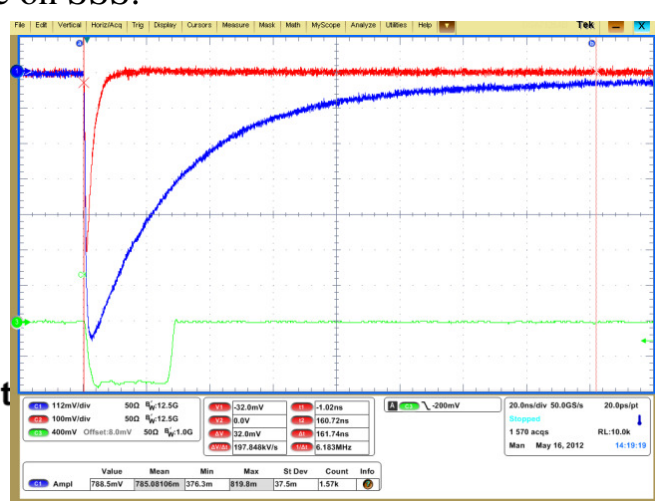

Fig. 3. MPPC. $3 \times 3 \mathrm{~mm}^{2}$. Red and blue traces - with and without clipping capacitance. Influence of the SiPM structure on SPTR was observed. A picture of the electric field distribution for the shallow junction SiPM produced by STM is shown in Fig. 4. For N on P junction the $n+$ side of the silicon faces the light. One can see that if a photon absorbs close to the SiPM surface then the originated carriers will be holes. Likewise, the carriers will be electrons if the photon is absorbed deep into the silicon. The absorption length is about $100 \mathrm{~nm}$ for the $405 \mathrm{~nm}$ photon (blue light PiLas head) and 4 um for the $635 \mathrm{~nm}$ (red light PiLas head).

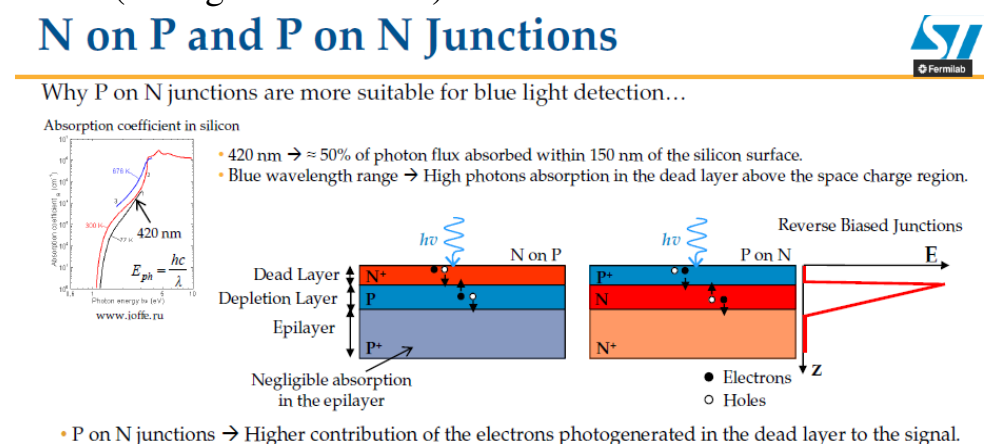

Fig. 4. Schematic view of SiPMs. N on P and P on N structures. 
Thus, blue photons produce mostly holes, which travel to the high electric field and eventually develop an avalanche ( $\mathrm{N}$ on $\mathrm{P}$ structure). The red photons produce mostly electrons traveling into the high field from the opposite direction. The mobility of holes in silicon's electric field is about 3 times less than for electrons, but the holes path length is 40 times less. So the combined time spread of carriers originated by blue photons should be about one order of magnitude less than that originated by red photons. This could explain why the SPTR is better for the blue light, Fig. 5.

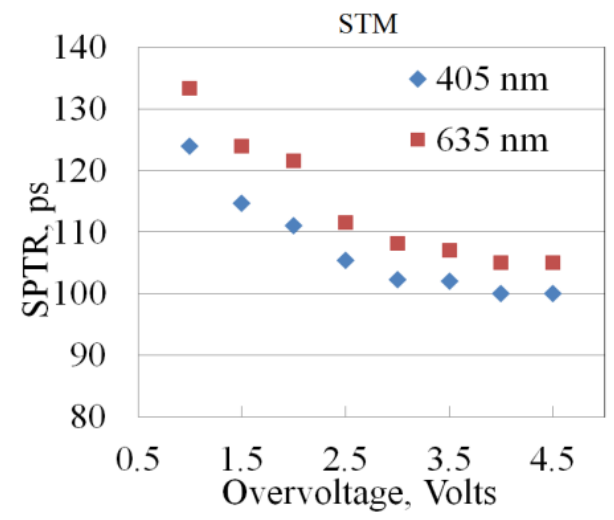

Fig. 5. STM, $3.5 \times 3.5 \mathrm{~mm}^{2}$, pixel size $50 \times 50 \mathrm{um}^{2}, 4,900$ pixels, $\mathrm{N}$ on $\mathrm{P}$ structure.

The magnitude of the velocity of carriers inside of silicon is about 1-10 ns per $300 \mathrm{um}$, depending on the electric field applied, silicon impurity and temperature. According to a rough estimation, a spread on the order of a few microns of travel distance could provide $100 \mathrm{ps}$ of the corresponding time spread. This simple picture does not take into consideration the time jitter due to an avalanche development, lateral avalanche size, but only considers the initial carrier's time spread. Nevertheless, this naïve model describes reasonably the data obtained for the $\mathrm{N}$ on P STM. For the STM with P on N structure the p+ side faces the light. The electrons are the carriers for the $405 \mathrm{~nm}$, and the holes are for the $635 \mathrm{~nm}$ in this case. We found almost no significant SPTR difference for the $3.5 \times 3.5 \mathrm{~mm}^{2}$ STMs for $405 \mathrm{~nm}$ and $635 \mathrm{~nm}$ illumination, $\sim 10 \%$ at most. We also do not see big SPTR difference for the STMs of $1 \times 1 \mathrm{~mm} 2$ and $3.5 \times 3.5 \mathrm{~mm} 2$, (same cell size). SPTR is at the level of $50-65$ ps for both size, Fig. 6, 7. All SiPMs show the SPTR improvement with the bias voltage increase, due to the avalanche probability increase.
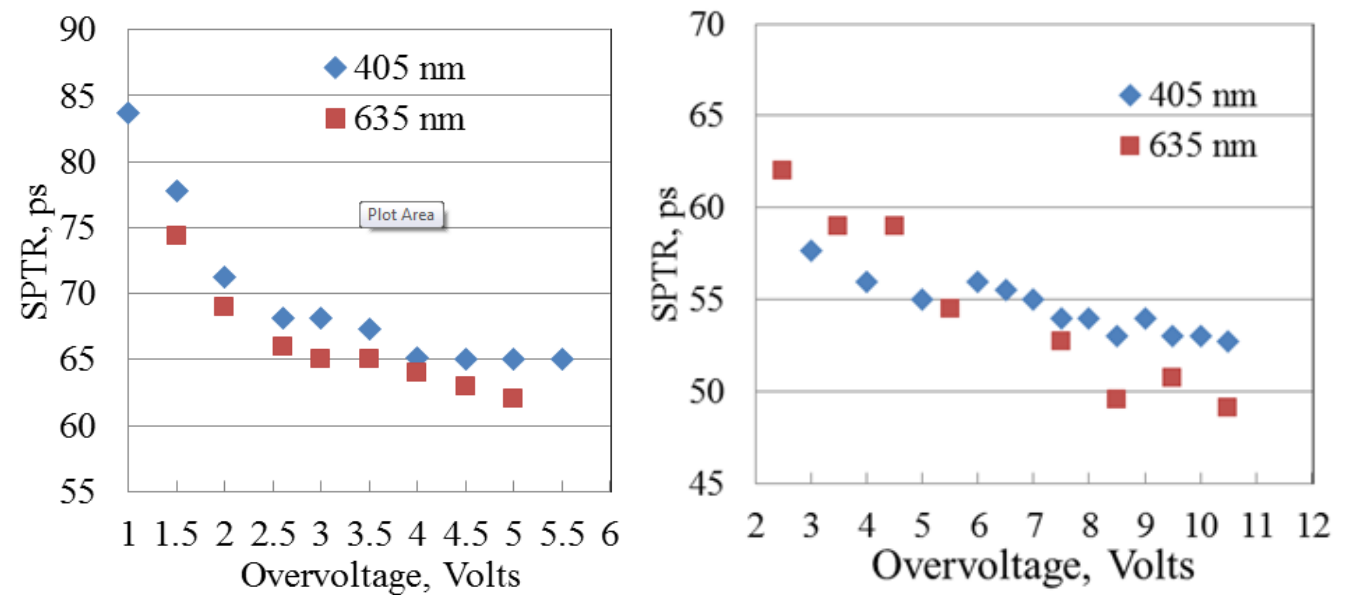
Fig. 6, left: SPTR for STM, P on N, 3.5x3.5 mm², 3,600 pixels, 58 um pitch. Fig. 7, right: STM, $\mathrm{P}$ on $\mathrm{N}, 1 \mathrm{x} 1 \mathrm{~mm}^{2}, 324$ pixels, 58 um pitch.
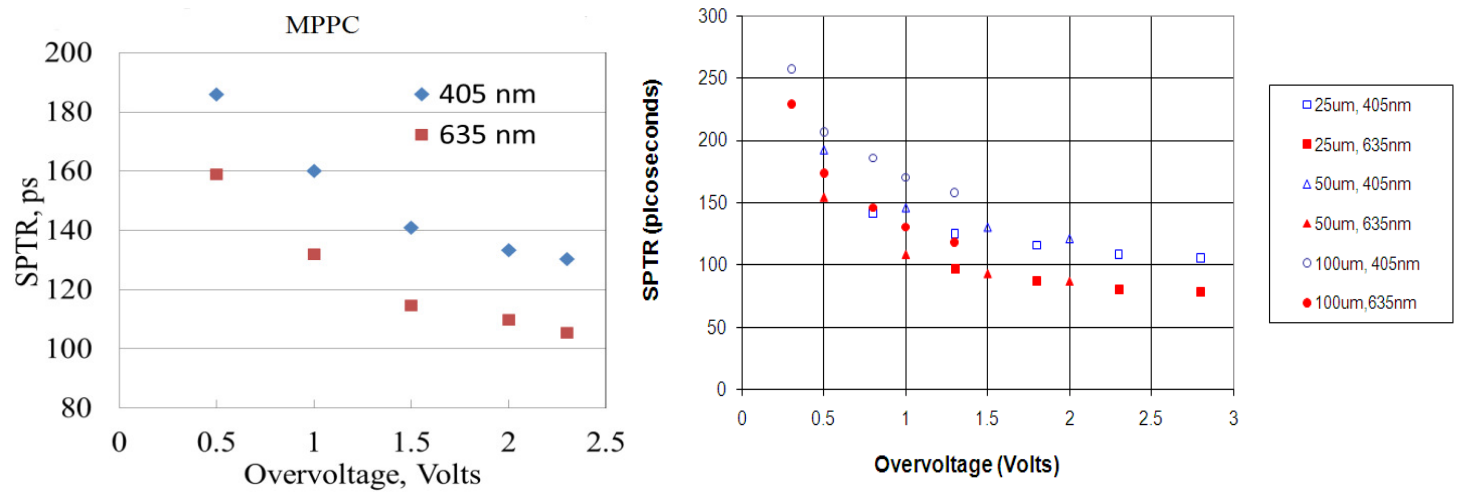

Fig. 8, left, SPTR for MPPC-S10362-33-050C, 3x3 mm². Fig. 9, right, SPTR for MPPC-S10362-11-025P，MPPC-S10362-11-050P，MPPC-S10362-11-100P， all 1x1 $\mathrm{mm}^{2}$.

Fig 8, 9 present SPTRs for MPPC, 3x3 mm2, and 1x1 mm2 for different cell size. Fig. 9 shows some SPTR improvement with smaller SiPM pixel size. About 10 times capacitance increases for $3.5 \times 3.5 \mathrm{~mm}^{2}$ size in comparison with $1 \times 1 \mathrm{~mm}^{2}$, should lead to significant SPTR worse as result. But it was not observed experimentally. Also no significant shape difference (especially $\mathrm{T}_{\mathrm{r}}$ ) observed when the clipping capacitance used. The STM single photoelectron clipped signals presented in Fig. 10.

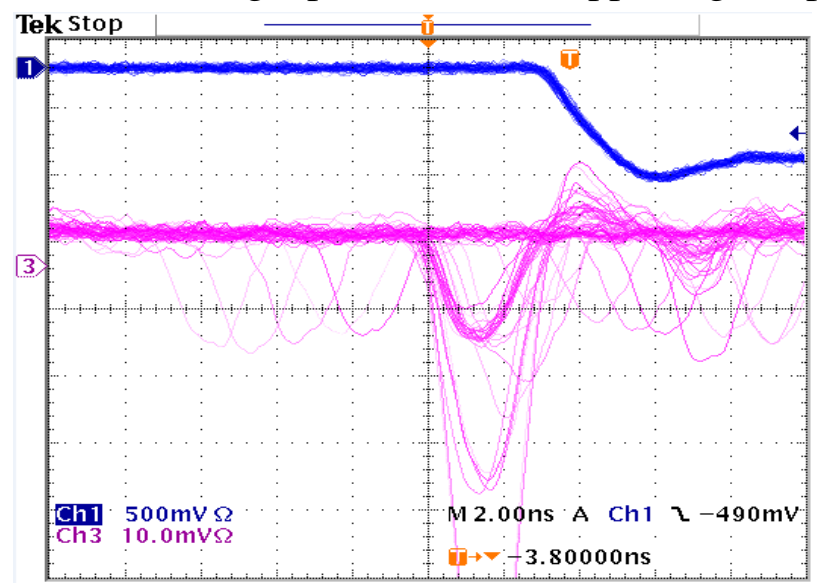

Fig. 10. Trace of single photoelectron. STM, 3.5x3.5 mm², 3,600 cells, P on N, 58 um pitch, 2.5V OV, $10 \mathrm{pF}$ clipping capacitance.

The signal's shape is almost the same for STM $1 \times 1 \mathrm{~mm} 2$, but noise is lower. Nevertheless, we can conclude that increased single photoelectron noise for $3.5 \times 3.5 \mathrm{~mm} 2$ does not affect the SPTRs significantly. Almost the same SPTR (for about one order larger SiPM size) approves very good cells uniformity. SPTR values for the MPPC-S10362-33-050C measured earlier were considerably larger. We use the same setup for the SPTR measurements which reproduce our old results. We can attribute these earlier results to the experimental MPPC samples, which were tested. 
Table of some SiPMs parameters.

\begin{tabular}{|c|c|c|c|c|c|c|c|c|}
\hline SiPMs & $\begin{array}{l}\text { Area, } \\
\mathrm{mm}^{2}\end{array}$ & $\begin{array}{l}\text { PDE, \%, } \\
430 \mathrm{~nm}\end{array}$ & $\begin{array}{l}\text { SPTR, } \\
\text { ps }\end{array}$ & $\begin{array}{l}\text { BV, } \\
\text { V }\end{array}$ & $\begin{array}{l}\mathrm{OV}, \\
\mathrm{V}\end{array}$ & Gain & $\begin{array}{l}\text { Pixels } \\
\text { amount }\end{array}$ & $\begin{array}{l}\text { Pixel, } \\
\text { um }^{2}\end{array}$ \\
\hline STM, & $\begin{array}{c}3.5 \times 3.5 \\
1 \times 1\end{array}$ & $31, \max$ & $\begin{array}{l}60-65 \\
50-53\end{array}$ & 28 & 4.5 & $\sim 10^{6}$ & $\begin{array}{r}3,600 \\
324\end{array}$ & $\begin{array}{l}60 \times 60 \\
60 \times 60\end{array}$ \\
\hline MPPC & $\begin{array}{l}3 \times 3 \\
1 \times 1\end{array}$ & $\begin{array}{l}\text { Up to } \\
50, \max ^{*}\end{array}$ & $\begin{array}{l}107-130 \\
80-120\end{array}$ & 70 & 2.3 & $\sim 10^{6}$ & $\begin{array}{r}3,600 \\
400\end{array}$ & $\begin{array}{l}50 \times 50 \\
50 \times 50\end{array}$ \\
\hline
\end{tabular}

*include effects of optical cross talks and after pulsing.

\subsection{Test beam results}

The MPPC with quartz radiator of $30 \mathrm{~mm}$ long were irradiated by $120 \mathrm{GeV}$ proton beam at normal incidence. The obtained time resolution for MPPCs is $14 \mathrm{ps.} \mathrm{Soft} \mathrm{pulse}$ height cuts applied. About the same result was obtained when we used DRS4 (Domino Ring Sampler). This is important because DRS4 has 200 ps/sample. 14.3 ps time resolution obtained with STMs and DRS4 readout, Fig. 11.
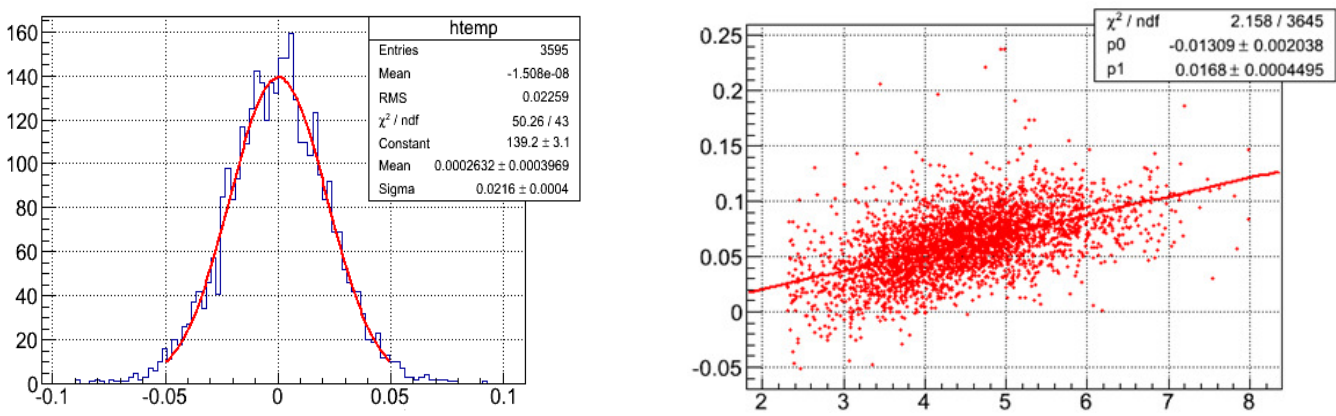

Fig. 11. Test beam result. Conditions: DRS4 readout, 2 STMs with $30 \mathrm{~mm}$ long quartz radiator, both along the beam line, $120 \mathrm{GeV}$ protons, normal incidence, TR is $\sim 14 \mathrm{ps,} \mathrm{about} \mathrm{the}$ same as with Ortec readout, left. Slew pulse height correction applied, right.

DRS4 principle is based on sampling and storing an incoming signal in an array of capacitors, Switch Capacitor Array (SCA), waiting for (selective) readout and digitization. DRS4 is capable to digitize 4 input channels at sampling rates 5 Giga-samples per second (GSPS, 200ps/sample). Individual channel depth is 1024 bins. BW is up to $850 \mathrm{MHz}$. DRS4 noise floor $\sim 1 \mathrm{mV} / 50$. DRS4 can replace old classic TDC, ADC traditional readout. PH and TR can be measured by the same unit.

\section{References}

[1] A. Ronzhin et al.,Test of timing properties of silicon photomultipliers, NIM, A616, 2010, 38-44. [2] A. Ronzhin et al.,Development of a 10 ps level time of flight system in the Fermilab Test beam facility, NIM, A623, 2010, 931-941.

[3] A. Ronzhin et al.,Waveform digitization for high resolution timing detectors with silicon photomultipliers, NIM, A668, 2012, 94-97. 